\title{
ESTILOS DE APRENDIZAJE Y SATISFACCIÓN FAMILIAR EN UN GRUPO DE ESTUDIANTES DE ALTO Y BAJO RENDIMIENTO DE UNA UNIVERSIDAD PRIVADA DE LIMA
}

\section{Learning styles and family satisfaction in a group of high and low performance students from a private university in Lima}

Jenny Quezada Zevallos*, Herbert Robles Mori** Universidad Femenina del Sagrado Corazón

\begin{abstract}
Resumen
La presente investigación es de tipo descriptivo comparativo, tiene el objetivo de describir y comparar el estilo de aprendizaje y el nivel de satisfacción familiar en un grupo de estudiantes de alto y bajo rendimiento de una universidad privada de Lima. La muestra de tipo no probabilístico, muestreo disponible, conformado por un total de 90 estudiantes, de las cuales 28 presentan Bajo rendimiento académico y 62 Alto rendimiento académico. Los instrumentos utilizados son: El Inventario de preferencias (Estilos de aprendizaje) de Katlen Buttler (1988) y la Escala de satisfacción familiar por adjetivos. Barraca, J \& Lópéz-Yarto, L. (2000). Con respecto a los estilos de aprendizaje el estilo de aprendizaje Abstracto Secuencial es el que se halla más desarrollado en toda la muestra estudiada y el estilo de aprendizaje Concreto Secuencial, en ambos grupos se concentran en un nivel Medio y Alto, siendo este estilo de aprendizaje el de mayor preferencia del grupo de estudio. El Total de Satisfacción familiar de ambos grupos presentan mayoritariamente un nivel Bajo $(82,1 \%$ y $82,1 \%$ respectivamente).
\end{abstract}

Palabras clave: Estilos de aprendizaje, satisfacción familiar, rendimiento académico.

\begin{abstract}
The present investigation is of a comparative descriptive type, with the objective of describing and comparing the learning style and the level of family satisfaction in a group of high and low performance students of a private university in Lima. The non-probabilistic sample, available sampling, made up of a total of 90 students, of which 28 have low academic performance and 62 high academic performance. The instruments used are: Katlen Buttler's Preference Inventory (Learning Styles) (1988) and the Family Satisfaction Scale for Adjectives. Barraca, J \& LópézYarto, L. (2000). With respect to learning styles, the Sequential Abstract learning style is the one that is most developed in the entire sample studied and the Sequential Concrete learning style, in both groups they are concentrated at a Medium and High level, this learning style being the most preferred of the study group. The Total Family Satisfaction of both groups mostly presents a Low level ( $82.1 \%$ and $82.1 \%$ respectively).
\end{abstract}

Keywords: Learning styles, family satisfaction, academic performance.

\footnotetext{
* Psicóloga. jennyquezadaz@unife.pe. ORCID: https://orcid.org/0000-0002-1408-241X

** Psicólogo.hrobles@unife.edu.pe. ORCID: https://orcid.org/0000-0002-5937-5937
} 


\section{INTRODUCCIÓN}

Esta investigación surge como un intento de obtener respuesta al complejo fenómeno del rendimiento académico universitario, partimos de que en este fenómeno de carácter pluricausal, algunos son propios de la persona como su personalidad, autoestima, hábitos de estudio, estilos de aprendizaje, etc, otros de origen contextual como el funcionamiento de la familia, las relaciones interpersonales, la situación sociocultural, etc..

En el presente estudio hemos seleccionado como variable personal, el estilo de aprendizaje y como variable contextual la satisfacción familiar.

\section{ANTECEDENTES}

Las investigaciones revelan que el rendimiento académico es multicausal y complejo, planteándose una interacción de múltiples factores sociales, personales, institucionales y académicos, al respecto Villarroel, V. (2011), Uribe, H.; Soares, N.; Omar, A.; Urteaga, A. (2010) informan correlaciones significativas entre autoconcepto, autoestima y rendimiento académico. En el estudio de Uribe, $\mathrm{H}$, se utiliza el concepto de capital cultural, social y humano los cuales correlacionan con autoimagen. Siendo importante señalar que el concepto de capital social implica las características socioambientales de la familiar, en especial las dimensiones estabilidad, desarrollo y relaciones.

Los estudios sobre la influencia de la familia en el rendimiento académico son variados así Luna, A. (2011), halló efecto de la frecuencia de conflictos sobre la satisfacción familiar en el grado de cohesión familiar, mientras que Guevara, E.; Jaramillo, R.; Tovar, S. (2013) informan no hallar influencia entre la estructura familiar (estrato socioeconómico, nivel de educación de los padres, conformación familiar y estilos parental) y el rendimiento académico, Herrera, L.; Rivera, M. (2011) informa correlación significativa entre malestar psicológico y percepción de pobre apoyo familiar.

Los hallazgos con respecto a la relación entre estilos de aprendizaje y rendimiento son contradictorios, así Bacon, Donald R \& Hartley, Steven W. (2015); Brunton, (2015) reportan no hallar relación entre dichas variables, otros informan correlación entre dichas variables, Esguerra, G.; Guerrero, P. (2010) entre los modos de aprendizaje de los estilos activo y reflexivo y rendimiento académico.

Otros estudios se han focalizado en el nivel socioeconómico de los estudiantes como predictores en el promedio ponderado y nota de admisión, al respecto Garbanzo, G. (2013) en un estudio con estudiantes con beca y sin beca, informa no hallar diferencias en los indicadores de satisfacción universitaria, hábitos de estudio, apoyo en los estudios universitarios, satisfacción con los profesores y nivel socioeconómico.

Se han realizado estudios sobre estilos de aprendizaje y su relación con múltiples aspectos del aprendizaje, así la relación entre dominancia cerebral y especialidad profesional (Segarra, Estrada, Monferrer, 2015), expectativas de superación de curso y elección de carrera relacionada con el estilo de aprendizaje (Antelm, Gil-López, Cacheiro-González, 2015; Terregrossa, Englander, Zhaobo, 2015; Chen Kang Lee \& Sidhu, Manjit Singh, 2015), el estilo de aprendizaje preferente en estudiantes de medicina (Shankar, Balasubramanium, Dwivedi, Ramireddy, Barton, 2014; Nuzhat, A. , Salem, O., Hamdan, N., Ashour, N., 2013), estilo de aprendizaje preferente en estudiantes de trabajo social (Williams, B., Brown, T., Etherington, J., 2013), diferencias de estilo de aprendizaje de acuerdo al sexo ( Vasquez, Noriega, García, 2013), estilos de aprendizaje y rendimiento académico en evaluaciones finales (Tan \& Laswad, 2015).

\section{ESTILOS DE APRENDIZAJE}

El estilo de aprendizaje se refiere a las diferencias individuales en la manera de captar, organizar y recordar la información. Algunas de estas maneras responden a disposiciones naturales y otras son resultados de las experiencias, exigencias del medio ambiente y aprendizajes de la persona.

Schmeck (1988) considera que el estilo de aprendizaje es una predisposición a utilizar una estrategia particular de aprendizaje, al margen de las demandas específicas; y la estrategia de aprendizaje 
es un conjunto de actividades de procesamiento de información que se utilizan para mejorar el aprendizaje.

Kogan (1976) Witkin (1969) (referidos por Martínez, Quezada, Sassone y Huarachi (2001)) consideran que los estilos cognitivos son diferencias cognitivas individuales asociadas con otras dimensiones no cognitivas de la personalidad, como motivaciones e intereses, sentimientos y actitudes.

Por otra parte es importante considerar que un estilo de aprendizaje solo indica su preferencia más frecuente, considerándose además para Verlee (1986) ) (referidos por Martínez, Quezada, Sassone y Huarachi (2001)) que las modalidades de pensamiento son también modalidades de aprendizaje, por las que éstas podrían clasificarse en visual, auditiva y cenestésico.

Consideramos que el concepto de estilo de aprendizaje conceptualiza las diferencias individuales distintivas y consistentes en la forma como la información es percibida, procesada y organizada

Kathleen Butler (1988) reconoce las Habilidades perceptivas: Concreta y Abstracta

- Los alumnos de estilo concreto registran información recibida por sus sentidos de vista, olfato, tacto, gusto y oído. Ven las cosas de una manera tangible, fáctica y literal. Se interesan por los productos.

- Los alumnos de estilo abstracto prefieren estudiar las relaciones y las ideas no visibles. Usan la intuición y la imaginación

Por otra parte, señala dos tipos de habilidades de ordenamiento: Secuencial y Disperso.

- Los alumnos de estilo secuencial organizan la información un paso a la vez. Usan la intuición y la imaginación.

- Los alumnos de estilo aleatorio son espontáneos. Utilizan variedad de opciones.

Combinando los dos tipos de Habilidades perceptivas y los dos tipos de Habilidades de ordenamiento, describe 4 estilos de aprendizaje:

- Estilo de aprendizaje concreto secuencial

- Estilo de aprendizaje abstracto secuencial

- Estilo de aprendizaje concreto disperso

- Estilo de aprendizaje abstracto disperso

Estilo de aprendizaje concreto secuencial: Los alumnos de estilo concreto registran información recibida por sus sentidos de vista, olfato, tacto, gusto y oído. Ven las cosas de una manera tangible, fáctica y literal y al ser secuenciales organizan la información un paso a la vez.

\section{Características del Estilo de aprendizaje concreto secuencial}

\begin{tabular}{|l|l|}
$\begin{array}{l}\text { Habilidades } \\
\text { Aprenden paso a paso } \\
\text { Tienen Planes }\end{array}$ & $\begin{array}{l}\text { Aprende mejor cuando } \\
\text { En ambiente ordenado/ callado } \\
\text { Prefieren una dirección exacta } \\
\text { Sigue direcciones }\end{array}$ \\
$\begin{array}{l}\text { Cuando la información es consistente y eficiente } \\
\text { Cuando es reforzado por el aprendizaje } \\
\text { Cuando puede analiza las ideas de una manera práctica } \\
\text { Son prácticos }\end{array}$ & Cuando puede utilizar la imaginación. \\
Prefieren los hechos & Como se estira el estilo \\
\hline Retos & Viendo el "bosque" \\
Aprender a escoger temas & Expresando sus sentimientos \\
Practicar nuevas estrategias & Considerando el proceso \\
Interpretar ideas abstractas & No dando la primera respuesta \\
Cuando hay puntos de vista distintos & Pidiendo explicación del pensamiento de otros. \\
Haciendo preguntas de qué pasaría si ...? & \\
\hline
\end{tabular}


Estilo de aprendizaje concreto disperso: Los alumnos de estilo concreto registran información recibida por sus sentidos de vista, olfato, tacto, gusto y oído. Ven las cosas de una manera tangible, fáctica y literal. y al ser dispersos presentan un estilo aleatorio son espontáneos, utilizan una variedad de opciones

Características del estilo de aprendizaje Concreto Disperso

\begin{tabular}{|c|c|}
\hline $\begin{array}{l}\text { Habilidades } \\
\text { Experimenta para encontrar respuestas. } \\
\text { Son independientes } \\
\text { Considera soluciones } \\
\text { Toma riesgos calculados } \\
\text { Investiga ¿porqué? } \\
\text { Alto grado de curiosidad. } \\
\text { Utiliza variedad de opciones }\end{array}$ & $\begin{array}{l}\text { Aprende mejor cuando } \\
\text { Trata nuevos abordajes para resolver problemas. } \\
\text { En situaciones competitivas } \\
\text { Creando sus propias respuestas. } \\
\text { Usando métodos de ensayo. } \\
\text { Actividades de lluvia de ideas. }\end{array}$ \\
\hline $\begin{array}{l}\text { Retos } \\
\text { Cuando se le limita el tiempo. } \\
\text { Finalizar productos } \\
\text { Seleccionar una respuesta } \\
\text { Mantener registros detallados. } \\
\text { Ser dirigido solamente a productos }\end{array}$ & $\begin{array}{l}\text { Como se estira el estilo } \\
\text { Aprender a priorizar. } \\
\text { Usar técnicas de autocontrol. } \\
\text { Delegar responsabilidades } \\
\text { Aceptar ideas de otros. } \\
\text { Preguntar acerca de cómo } \\
\text { Conocer cuando es imposible un cambio }\end{array}$ \\
\hline
\end{tabular}

Estilo de aprendizaje abstracto secuencial: Los alumnos de estilo abstracto prefieren estudiar las relaciones y las ideas no visibles y al ser secuenciales organizan la información un paso a la vez.

Características del Estilo de aprendizaje Abstracto Secuencial

\section{Habilidades}

Debate puntos de vista.

Organiza ideas

Adquiere información / la analiza.

Piensa de manera estructurada.

Paciente con personas que aprenden despacio.

Juzga valores e importancia.

Investiga

\section{Retos}

Expresar emociones constructivamente.

Descubrir formas distintas de aprender

Juzgando / simulando

Riesgos

Ser criticado

\section{Aprende mejor cuando}

Tiene referencias de viajes, aventuras, etc.

Está seguro

Sigue procedimientos

Tiene tiempo para aprender el material.

Pregunta ¿por qué es así?

Utiliza puntos anotados

Es respetada por su inteligencia.

\section{Como se estira el estilo}

Ver el humor de las cosas

Tomar el tiempo para "oler la res"

Poner calificaciones en perspectiva

Explorar sentimientos personales

Se preocupa menos

No debate/ argumentar 
Estilo de aprendizaje abstracto disperso: Los alumnos de estilo abstracto prefieren estudiar las relaciones y las ideas no visibles y al ser dispersos presentan un estilo aleatorio son espontáneos, utilizan una variedad de opciones

\begin{tabular}{|c|c|}
\hline Habilidades & Aprende mejor cuando \\
\hline Reflexiona acerca de sentimientos & Puede trabajar y compartir con otros. \\
\hline Son flexibles & Da atención personal. \\
\hline Información personalizada & Balanceando trabajo con actividades sociales. \\
\hline Aprecia el arte, etc. & Usa expresión artística. \\
\hline Usa imaginación para crear. & Se comunica libremente con otros. \\
\hline Ve el bosque & En atmósferas competitivas. \\
\hline \multicolumn{2}{|l|}{ Interpreta sentimientos e ideas } \\
\hline Retos & Como se estira el estilo \\
\hline Repetir & Ver los árboles en el bosque \\
\hline Memorizar & Reacciona menos emocionalmente a demandas, a enunciados \\
\hline Llamar exactamente de la misma forma & Mira antes de reaccionar \\
\hline Etapa por etapa & Presta atención a detalles importantes \\
\hline Tiempo límite & Incluye información objetiva al tomar decisiones. \\
\hline Material secuenciado, proceso & \\
\hline
\end{tabular}

\section{SATISFACCION FAMILIAR}

Las investigaciones sobre familia y desempeño académico informan relación entre dichas variables (Guzmán \& Pacheco, 2014; Cheng, Ickes, Verhofstadt, 2012), a diferencia de Guevara, E. P.; Jaramillo R. yTovar, S. (2013) quienes informan no hallar influencia de los factores familiares en el rendimiento académico; Jiménez, Aguado, Álvarez, Gil, y Jiménez, (2002) en la descripción de las características sociológicas de los alumnos del Premio Nacional de Bachillerato hallaron que los alumnos son procedentes de la clase media y media alta, de familias estables y maduras. Guevara, Jaramillo, y Tovar Paredes, S. (2013) informan no hallar relación entre promedio académico y nivel educativo del padre y/o de la madre, ni tampoco con la estructura familiar.

La Satisfacción familiar se entiende como un conjunto de sentimientos que se evidencian en las personas al estar con su familia, que son el resultado de una serie de interacciones positivas, nutritivas y gratificantes que se establecen y se mantienen al interior de ella. Estas relaciones son vividas de manera propia y única en cada miembro de la familia.
En el trabajo realizado por Quezada J.; Zavala E. Lenti M. (2015) proponen las siguientes dimensiones de la Satisfacción familiar:

- Amor familiar

- Sentido de pertenencia a la familia

- Sostenimiento familiar/holding

- Felicidad

- Confianza

- Alegría

- Tranquilidad y confianza

A continuación, la definición de cada una de estas dimensiones de la Satisfacción familiar.

Amor familiar: Sentimiento surgido entre los miembros de un sistema familiar, que los ayuda a conectarse, relacionarse y enlazarse, otorgando reconocimiento al existir del "otro" en sus propias vidas.

Sentido de pertenencia a la familia: Conjunto de acciones, valoraciones, sentimientos y reconocimientos que experimentan los individuos que conforman un sistema familiar y del cual se consideran parte activa. 
Sostenimiento familiar/holding: Proceso que realiza el sistema adulto, generalmente los padres, en función de sus hijos u otros miembros de la familia que así lo requiriesen, con la finalidad de brindar el necesario apoyo en favor del desarrollo integral de sus miembros.

Felicidad: Es el estado afectivo de satisfacción y regocijo que experimenta subjetivamente el individuo al alcanzar sus expectativas y logros o por el bienestar que vive al sentirse inmerso en un medio que lo retroalimenta de manera positiva y afectiva.

Confianza: Se refiere a la creencia y seguridad que una persona desarrolla respecto de sí misma, a una situación o a la forma de actuar de otra persona. Ésta puede generarse de una manera consciente a partir de situaciones particulares y/o experiencias vividas en cada individuo. Es una vivencia única e individual que se relaciona con un sentimiento de tranquilidad focalizado en las expectativas de una acción futura y que puede reforzarse o debilitarse en función de las acciones de las otras personas o de las circunstancias que enfrenta.

Alegría: Es una vivencia afectiva de placer y satisfacción que se asocia a una fuerza impulsora, de entrega y apertura hacia los demás y que puede percibirse en toda persona que la experimente a través de su apariencia, su lenguaje, en sus acciones constructivas y positivas, reflejando un estado de armonía y felicidad.

Tranquilidad y confianza: Son sentimientos positivos que reflejan un estado de bienestar, aceptación y seguridad, que se manifiesta cuando la persona vive momentos significativos y que le brinda un sentimiento de satisfacción, plenitud y equilibrio interior

Los objetivos del estudio son:

1. Describir y comparar el estilo de aprendizaje en un grupo de estudiantes de alto y bajo rendimiento de una universidad privada de Lima.

2. Describir y comparar el nivel de satisfacción familiar en un grupo de estudiantes de alto y bajo rendimiento de una universidad privada de Lima.

\section{MÉTODO}

Estudio de tipo descriptivo comparativo

\section{MUESTRA}

Población conformada por estudiantes universitarias de alto rendimiento (122) y bajo rendimiento (124), de 4 escuelas profesionales de una universidad privada.

Muestra de tipo no probabilístico y para conformación de la misma se utilizó el muestreo disponible, 90 estudiantes, de las cuales 28 presentan Bajo rendimiento académico y 62 Alto rendimiento académico, contándose con el Consentimiento Informado de todo el grupo de estudio.

\section{INSTRUMENTOS}

Los instrumentos utilizados son:

Inventario de preferencias (Estilos de aprendizaje) de Katlen Buttler (1988), el cual está conformado por 40 reactivos frente a cada uno de los cuales se responde con una escala de Likert de 4, siendo (4 ) lo que más me gusta y (1) lo que menos me gusta.

Escala de Satisfacción Familiar por adjetivos (ESFA) de J. Barraza y L. López (2010). La prueba está conformada por 27 items, cada uno formado por una pareja de adjetivos antónimos, correspondiendo la estructura a un diferencial semántico. La consistencia interna en la muestra de mujeres, informada por los autores se logró a través de la prueba Alfa de Cronbach (0.9771) y "Dos mitades" (0.9628). La estabilidad temporal se evaluó a través de la correlación test-retest $(0,7941 *)$. La validez de constructo se realizó a través del análisis factorial, apareciendo 3 factores con un autovalor mayor que 1.00 , siendo el I factor el que explica por sí sólo el $62,3 \%$.

Para esta investigación se elaboraron los percentiles de ambas pruebas con una muestra de 41 personas.

En la tabla 1, se puede observar los valores percentiles para la variable preferencias. 
Tabla 1

Percentiles para la variable Preferencias (Estilos de aprendizaje) $(N=41)$.

\begin{tabular}{|c|c|c|c|c|c|}
\hline & & Concreto-Disperso & Abstracto-Disperso & Abstracto-Secuencial & Concreto-Secuencial \\
\hline \multicolumn{2}{|l|}{ Media } & 20,5122 & 18,0244 & 25,5610 & 17,1220 \\
\hline \multicolumn{2}{|l|}{ Mediana } & 21,0000 & 18,0000 & 25,0000 & 17,0000 \\
\hline \multirow[t]{21}{*}{ Percentiles } & 1 & 13,0000 & 11,0000 & 15,0000 & 9,0000 \\
\hline & 5 & 13,0000 & 12,0000 & 18,1000 & 10,1000 \\
\hline & 10 & 15,4000 & 12,0000 & 20,2000 & 11,2000 \\
\hline & 15 & 17,0000 & 13,3000 & 21,0000 & 13,3000 \\
\hline & 20 & 17,4000 & 14,4000 & 21,4000 & 14,0000 \\
\hline & 25 & 18,0000 & 15,0000 & 22,5000 & 14,0000 \\
\hline & 30 & 19,0000 & 15,0000 & 23,6000 & 14,0000 \\
\hline & 35 & 19,0000 & 16,0000 & 24,0000 & 14,7000 \\
\hline & 40 & 19,8000 & 17,6000 & 24,0000 & 16,0000 \\
\hline & 45 & 20,0000 & 18,0000 & 25,0000 & 16,9000 \\
\hline & 50 & 21,0000 & 18,0000 & 25,0000 & 17,0000 \\
\hline & 55 & 22,0000 & 19,0000 & 26,0000 & 18,0000 \\
\hline & 60 & 22,0000 & 19,0000 & 26,0000 & 18,0000 \\
\hline & 65 & 22,3000 & 20,0000 & 27,3000 & 18,3000 \\
\hline & 70 & 23,0000 & 21,0000 & 28,4000 & 19,4000 \\
\hline & 75 & 23,0000 & 21,0000 & 29,0000 & 20,5000 \\
\hline & 80 & 23,0000 & 21,6000 & 29,6000 & 21,0000 \\
\hline & 85 & 24,0000 & 22,0000 & 30,0000 & 21,0000 \\
\hline & 90 & 24,0000 & 23,8000 & 30,8000 & 23,0000 \\
\hline & 95 & 26,8000 & 24,0000 & 32,9000 & 24,0000 \\
\hline & 99 & a más & a más & a más & a más \\
\hline
\end{tabular}

Escala de satisfacción familiar por adjetivos. Barraca, J \& Lópéz - Yarto, L. (2000)

En la tabla 2, se puede observar los resultados de la correlación total de elementos corregidos, el
Alfa si el elemento se ha suprimido; se observa que no se elimina ningún ítems, Sin embargo para estos resultados es necesario cambiar el sentido de la corrección de los siguientes Ítems: 1,3, 4, 6, 9, 10, 13, 17, 20, 22, 24, 23, 26. 
Tabla 2

Correlación, alfa de Cronbach, para los ítems de la Escala de Satisfacción Familiar, del estudio Piloto.

\begin{tabular}{|c|c|c|c|c|c|}
\hline \multicolumn{3}{|c|}{ Amor familiar } & \multicolumn{3}{|c|}{ Felicidad } \\
\hline Nro Items & $\begin{array}{c}\text { Correlación total } \\
\text { de elementos corregida }\end{array}$ & $\begin{array}{l}\text { Alfa si el elemento } \\
\text { se ha suprimido }\end{array}$ & Nro Items & $\begin{array}{l}\text { Correlación total } \\
\text { de elementos corregida }\end{array}$ & $\begin{array}{c}\text { Alfa si el elemento } \\
\text { se ha suprimido }\end{array}$ \\
\hline 2 &, 376 &, 833 & 1 &, 689 &, 891 \\
\hline 4 & ,474 & 824 & 3 & ,497 & 896 \\
\hline 5 & ,485 & ,825 & 6 & 207 & ,907 \\
\hline 8 &, 345 & ,835 & 7 & ,788 & 887 \\
\hline 13 & 312 & 841 & 9 & 841 & 889 \\
\hline 16 &, 589 & ,814 & 10 & ,442 & 898 \\
\hline 18 &, 593 & 817 & 11 &, 550 & 894 \\
\hline 21 & ,579 & 817 & 12 & ,395 & ,900 \\
\hline 24 & ,633 & ,812 & 14 &, 776 & 889 \\
\hline 25 & ,650 & ,810 & 15 & ,676 & 891 \\
\hline \multirow[t]{6}{*}{26} & ,708 & 803 & 17 &, 568 & 896 \\
\hline & & 19 & ,431 & 899 & \\
\hline & & 20 & 666 & 890 & \\
\hline & & 22 &, 580 & 893 & \\
\hline & & 23 &, 713 & ,888 & \\
\hline & & 27 & ,755 & 888 & \\
\hline
\end{tabular}

En la tabla 3, se puede observar los Alfa de Cronbach, para la escala de Satisfacción familiar, se aprecia que el Alfa más alto corresponde al total de la prueba con un valor de .739 .

Tabla 3

Alfa de Cronbach para las dimensiones de la Escala de Satisfacción Familiar.

\begin{tabular}{lc}
\hline Dimensión & Alfa de Cronbach \\
\hline Amor Familiar & .835 \\
Felicidad & .900 \\
Total & .932 \\
\hline
\end{tabular}

En la tabla 4, se puede observar los valores percentiles para la variable Satisfacción Familiar. 
Tabla 4.

Percentiles para la variable Satisfacción Familiar $(N=41)$

\begin{tabular}{|c|c|c|c|c|c|c|c|c|}
\hline & & Holding & Sentido Pertenecía & Amor Familiar & Alegría & Tranquilidad & Felicidad & Total \\
\hline \multirow{2}{*}{\multicolumn{2}{|c|}{$\begin{array}{l}\text { Media } \\
\text { Mediana }\end{array}$}} & 24,12 & 28,88 & 53,00 & 40,59 & 38,56 & 79,15 & 132,15 \\
\hline & & 25,00 & 29,00 & 54,00 & 40,00 & 40,00 & 82,00 & 134,00 \\
\hline \multirow[t]{21}{*}{ Percentiles } & 1 & 16,00 & 16,00 & 34,00 & 22,00 & 14,00 & 36,00 & 70,00 \\
\hline & 5 & 18,00 & 20,00 & 39,00 & 32,00 & 25,20 & 59,70 & 100,10 \\
\hline & 10 & 19,00 & 21,40 & 40,00 & 33,20 & 28,20 & 66,40 & 110,60 \\
\hline & 15 & 20,00 & 24,00 & 44,30 & 36,30 & 32,30 & 68,60 & 113,30 \\
\hline & 20 & 20,00 & 24,40 & 46,00 & 38,00 & 33,40 & 71,00 & 116,80 \\
\hline & 25 & 21,50 & 26,50 & 47,50 & 38,00 & 34,50 & 73,50 & 121,50 \\
\hline & 30 & 22,00 & 27,00 & 50,00 & 38,00 & 36,00 & 74,00 & 123,60 \\
\hline & 35 & 22,70 & 27,00 & 51,70 & 38,00 & 37,00 & 74,00 & 129,40 \\
\hline & 40 & 23,80 & 28,00 & 52,00 & 38,80 & 39,80 & 76,60 & 131,80 \\
\hline & 45 & 24,90 & 28,00 & 53,00 & 40,00 & 40,00 & 78,00 & 132,00 \\
\hline & 50 & 25,00 & 29,00 & 54,00 & 40,00 & 40,00 & 82,00 & 134,00 \\
\hline & 55 & 25,10 & 29,10 & 55,00 & 41,10 & 41,00 & 83,00 & 135,10 \\
\hline & 60 & 26,00 & 31,00 & 55,40 & 42,20 & 42,00 & 84,00 & 136,60 \\
\hline & 65 & 26,00 & 31,00 & 57,00 & 43,00 & 43,00 & 84,30 & 140,60 \\
\hline & 70 & 27,00 & 32,00 & 58,40 & 44,40 & 43,00 & 86,40 & 144,00 \\
\hline & 75 & 27,00 & 33,00 & 59,50 & 45,00 & 43,00 & 87,00 & 145,50 \\
\hline & 80 & 27,00 & 33,60 & 60,60 & 45,60 & 44,00 & 88,20 & 146,60 \\
\hline & 85 & 27,70 & 34,70 & 61,70 & 46,70 & 44,00 & 89,70 & 151,40 \\
\hline & 90 & 28,80 & 35,00 & 62,00 & 47,80 & 44,80 & 92,80 & 153,80 \\
\hline & 95 & 29,00 & 35,90 & 62,90 & 48,00 & 47,00 & 94,80 & 155,00 \\
\hline & 99 & a más & a más & a más & a más & a más & a más & a más \\
\hline
\end{tabular}

\section{RESULTADOS Y DISCUSIÓN}

Rendimiento académico y estilos de aprendizaje

En relación con el estilo de aprendizaje Concreto Disperso el mayor porcentaje en el grupo de rendimiento Bajo se encuentra en el nivel Medio en un
$50 \%$ a diferencia del grupo de rendimiento Académico Alto que se encuentra en el nivel Bajo (51.6\%), pudiendo deducirse que este estilo no caracteriza al grupo de rendimiento Alto. 
Tabla 5

Rendimiento académico y estilo de aprendizaje Concreto Disperso

\begin{tabular}{|c|c|c|c|c|c|c|}
\hline & & & \multicolumn{3}{|c|}{ Estilo de aprendizaje Concreto Disperso (E.A CD) } & \multirow[b]{2}{*}{ Total } \\
\hline & & & Bajo & Medio & Alto & \\
\hline \multirow[t]{8}{*}{ Rendimiento } & Bajo & Recuento & 11 & 14 & 3 & 28 \\
\hline & & $\%$ dentro de Rendimiento & $39,3 \%$ & $50,0 \%$ & $10,7 \%$ & $100,0 \%$ \\
\hline & & $\%$ dentro de E.A.CD & $25,6 \%$ & $35,9 \%$ & $37,5 \%$ & $31,1 \%$ \\
\hline & & $\%$ del total & $12,2 \%$ & $15,6 \%$ & $3,3 \%$ & $31,1 \%$ \\
\hline & Alto & Recuento & 32 & 25 & 5 & 62 \\
\hline & & $\%$ dentro de Rendimiento & $51,6 \%$ & $40,3 \%$ & $8,1 \%$ & $100,0 \%$ \\
\hline & & $\%$ dentro de E.A.CD & $74,4 \%$ & $64,1 \%$ & $62,5 \%$ & $68,9 \%$ \\
\hline & & $\%$ del total & $35,6 \%$ & $27,8 \%$ & $5,6 \%$ & $68,9 \%$ \\
\hline \multirow[t]{4}{*}{ Total } & & Recuento & 43 & 39 & 8 & 90 \\
\hline & & $\%$ dentro de Rendimiento & $47,8 \%$ & $43,3 \%$ & $8,9 \%$ & $100,0 \%$ \\
\hline & & $\%$ dentro de E.A.CD & $100,0 \%$ & $100,0 \%$ & $100,0 \%$ & $100,0 \%$ \\
\hline & & $\%$ del total & $47,8 \%$ & $43,3 \%$ & $8,9 \%$ & $100,0 \%$ \\
\hline
\end{tabular}

Con relación al estilo de aprendizaje Abstracto Disperso, tanto el grupo de rendimiento académico Bajo como Alto presentan los mayores porcentajes en el nivel Medio, con la diferencia que, en el grupo de

Tabla 6

Rendimiento académico y estilo de aprendizaje Abstracto Disperso

\begin{tabular}{|c|c|c|c|c|c|c|}
\hline & & & \multicolumn{3}{|c|}{ Estilo de aprendizaje Abstracto Disperso (E.A.AD) } & \multirow[b]{2}{*}{ Total } \\
\hline & & & Bajo & Medio & Alto & \\
\hline \multirow[t]{8}{*}{ Rendimiento } & Bajo & Recuento & 7 & 13 & 8 & 28 \\
\hline & & $\%$ dentro de Rendimiento & $25,0 \%$ & $46,4 \%$ & $28,6 \%$ & $100,0 \%$ \\
\hline & & $\%$ dentro de E.A.AD & $21,9 \%$ & $31,0 \%$ & $50,0 \%$ & $31,1 \%$ \\
\hline & & $\%$ del total & $7,8 \%$ & $14,4 \%$ & $8,9 \%$ & $31,1 \%$ \\
\hline & Alto & Recuento & 25 & 29 & 8 & 62 \\
\hline & & $\%$ dentro de Rendimiento & $40,3 \%$ & $46,8 \%$ & $12,9 \%$ & $100,0 \%$ \\
\hline & & $\%$ dentro de E.A.AD & $78,1 \%$ & $69,0 \%$ & $50,0 \%$ & $68,9 \%$ \\
\hline & & $\%$ del total & $27,8 \%$ & $32,2 \%$ & $8,9 \%$ & $68,9 \%$ \\
\hline \multirow[t]{4}{*}{ Total } & & Recuento & 32 & 42 & 16 & 90 \\
\hline & & $\%$ dentro de Rendimiento & $35,6 \%$ & $46,7 \%$ & $17,8 \%$ & $100,0 \%$ \\
\hline & & $\%$ dentro de E.A.AD & $100,0 \%$ & $100,0 \%$ & $100,0 \%$ & $100,0 \%$ \\
\hline & & $\%$ del total & $35,6 \%$ & $46,7 \%$ & $17,8 \%$ & $100,0 \%$ \\
\hline
\end{tabular}

De acuerdo a los resultados anteriores, las estudiantes de rendimiento académico Alto no se caracterizan por los estilos de aprendizaje Concreto Disperso y Abstracto Disperso, siendo el elemento común de ambos estilos la Dispersión, habilidad de ordenamiento que se refiere a alumnos de estilo aleatorio y espontáneos. Utilizan variedad de rendimiento académico Alto, un $40.3 \%$ presenta este estilo de aprendizaje en un nivel Bajo, por lo que se puede deducir que no lo caracteriza. opciones. Al respecto es importante considerar que las escuelas profesionales de procedencia de las estudiantes estimulan procesos de aprendizaje en los cuales la Producción Convergente es premiada con altos calificativos. 
En cuanto al estilo de aprendizaje Abstracto Secuencial, tanto el grupo de rendimiento Bajo (60.7\%) como el grupo de rendimiento Alto (50\%) se concentra en el nivel Medio, hallándose porcentajes significativos en el nivel alto, pudiendo deducirse que este estilo es que más desarrollado se halla en toda la muestra estudiada.

Tabla 7

Rendimiento académico y estilo de aprendizaje Abstracto Secuencial

\begin{tabular}{|c|c|c|c|c|c|c|}
\hline & & & \multicolumn{3}{|c|}{ Estilo de aprendizaje Abstracto Secuencial (E.A.AS) } & \multirow[b]{2}{*}{ Total } \\
\hline & & & Bajo & Medio & Alto & \\
\hline \multirow[t]{8}{*}{ Rendimiento } & Bajo & Recuento & 3 & 17 & 8 & 28 \\
\hline & & $\%$ dentro de Rendimiento & $10,7 \%$ & $60,7 \%$ & $28,6 \%$ & $100,0 \%$ \\
\hline & & $\%$ dentro de E.A.AS & $20,0 \%$ & $35,4 \%$ & $29,6 \%$ & $31,1 \%$ \\
\hline & & $\%$ del total & $3,3 \%$ & $18,9 \%$ & $8,9 \%$ & $31,1 \%$ \\
\hline & Alto & Recuento & 12 & 31 & 19 & 62 \\
\hline & & $\%$ dentro de Rendimiento & $19,4 \%$ & $50,0 \%$ & $30,6 \%$ & $100,0 \%$ \\
\hline & & $\%$ dentro de E.A.AS & $80,0 \%$ & $64,6 \%$ & $70,4 \%$ & $68,9 \%$ \\
\hline & & $\%$ del total & $13,3 \%$ & $34,4 \%$ & $21,1 \%$ & $68,9 \%$ \\
\hline \multirow[t]{4}{*}{ Total } & & Recuento & 15 & 48 & 27 & 90 \\
\hline & & $\%$ dentro de Rendimiento & $16,7 \%$ & $53,3 \%$ & $30,0 \%$ & $100,0 \%$ \\
\hline & & $\%$ dentro de E.A.AS & $100,0 \%$ & $100,0 \%$ & $100,0 \%$ & $100,0 \%$ \\
\hline & & $\%$ del total & $16,7 \%$ & $53,3 \%$ & $30,0 \%$ & $100,0 \%$ \\
\hline
\end{tabular}

En cuanto al estilo de aprendizaje Concreto Secuencial, en ambos grupos hallamos que se concentran en un nivel Medio y Alto, siendo el estilo de aprendizaje de mayor preferencia del grupo de estudio. En nuestra opinión dicho resultado se halla relacionado con el tipo de escuela profesional (Ciencias y Humanidades) de las cuales proceden las estudiantes y el estilo de evaluación que apunta hacia respuestas de tipo Producción Convergente.

Tabla 8

Rendimiento académico y estilo de aprendizaje Concreto Secuencial

\begin{tabular}{|c|c|c|c|c|c|c|}
\hline & & & \multicolumn{3}{|c|}{ Estilo de aprendizaje Concreto Secuencial (E.A.CS) } & \multirow[b]{2}{*}{ Total } \\
\hline & & & Bajo & Medio & Alto & \\
\hline \multirow[t]{8}{*}{ Rendimiento } & Bajo & Recuento & 6 & 13 & 9 & 28 \\
\hline & & $\%$ dentro de Rendimiento & $21,4 \%$ & $46,4 \%$ & $32,1 \%$ & $100,0 \%$ \\
\hline & & $\%$ dentro de E.A.CS & $42,9 \%$ & $27,1 \%$ & $32,1 \%$ & $31,1 \%$ \\
\hline & & $\%$ del total & $6,7 \%$ & $14,4 \%$ & $10,0 \%$ & $31,1 \%$ \\
\hline & Alto & Recuento & 8 & 35 & 19 & 62 \\
\hline & & $\%$ dentro de Rendimiento & $12,9 \%$ & $56,5 \%$ & $30,6 \%$ & $100,0 \%$ \\
\hline & & $\%$ dentro de E.A.CS & $57,1 \%$ & $72,9 \%$ & $67,9 \%$ & $68,9 \%$ \\
\hline & & $\%$ del total & $8,9 \%$ & $38,9 \%$ & $21,1 \%$ & $68,9 \%$ \\
\hline \multirow[t]{4}{*}{ Total } & & Recuento & 14 & 48 & 28 & 90 \\
\hline & & $\%$ dentro de Rendimiento & $15,6 \%$ & $53,3 \%$ & $31,1 \%$ & $100,0 \%$ \\
\hline & & $\%$ dentro de E.A.CS & $100,0 \%$ & $100,0 \%$ & $100,0 \%$ & $100,0 \%$ \\
\hline & & $\%$ del total & $15,6 \%$ & $53,3 \%$ & $31,1 \%$ & $100,0 \%$ \\
\hline
\end{tabular}


En síntesis:

- El estilo de aprendizaje Concreto Disperso y Abstracto Disperso no son de preferencia del grupo de rendimiento académico Alto, siendo el factor común la habilidad de ordenamiento de Dispersión, la cual se caracteriza porque su orden es de acuerdo con sus prioridades.

- Los estilos Concreto Secuencial y Abstracto Secuencial son los de mayor preferencia por todo el grupo de estudio, siendo el factor común la habilidad de ordenamiento de Secuencialidad, la cual se caracteriza porque su orden es por secuencia de pasos, de principio a fin.

\section{Rendimiento académico y satisfacción familiar}

El estudio de la relación entre rendimiento académico y satisfacción familiar se realizó considerando las dimensiones de: Holding, Sentido de pertenencia, Amor familiar, Alegría, Tranquilidad y Felicidad.

Tabla 9

Rendimiento académico y Holding
En relación con el rendimiento académico y la dimensión Holding, el grupo de rendimiento académico Bajo presenta el 50\% en un nivel Bajo, a diferencia del grupo de rendimiento académico Alto que presenta 58\% (acumulando los niveles Medio y Alto), pudiendo deducirse que el Holding es adecuado en dicho grupo.

El grupo estudiado se halla en el período entre finales de la adolescencia y adultez joven, viviendo la gran mayoría con sus padres, quienes sostienen emocional y económicamente a la estudiante, en tal sentido el Holding o sostenimiento familiar se constituye en el necesario apoyo en favor del desarrollo integral de sus miembros y al mismo tiempo en el espacio de conflictos padres- hijos y/o conflictos fraternales.

\begin{tabular}{|c|c|c|c|c|c|c|}
\hline & & & \multicolumn{3}{|c|}{ Holding } & \multirow[b]{2}{*}{ Total } \\
\hline & & & Bajo & Medio & Alto & \\
\hline \multirow[t]{8}{*}{ Rendimiento } & Bajo & Recuento & 14 & 9 & 5 & 28 \\
\hline & & $\%$ dentro de Rendimiento & $50,0 \%$ & $32,1 \%$ & $17,9 \%$ & $100,0 \%$ \\
\hline & & $\%$ dentro de Holding & $35,0 \%$ & $26,5 \%$ & $31,3 \%$ & $31,1 \%$ \\
\hline & & $\%$ del total & $15,6 \%$ & $10,0 \%$ & $5,6 \%$ & $31,1 \%$ \\
\hline & Alto & Recuento & 26 & 25 & 11 & 62 \\
\hline & & $\%$ dentro de Rendimiento & $41,9 \%$ & $40,3 \%$ & $17,7 \%$ & $100,0 \%$ \\
\hline & & $\%$ dentro de Holding & $65,0 \%$ & $73,5 \%$ & $68,8 \%$ & $68,9 \%$ \\
\hline & & $\%$ del total & $28,9 \%$ & $27,8 \%$ & $12,2 \%$ & $68,9 \%$ \\
\hline \multirow[t]{4}{*}{ Total } & & Recuento & 40 & 34 & 16 & 90 \\
\hline & & $\%$ dentro de Rendimiento & $44,4 \%$ & $37,8 \%$ & $17,8 \%$ & $100,0 \%$ \\
\hline & & $\%$ dentro de Holding & $100,0 \%$ & $100,0 \%$ & $100,0 \%$ & $100,0 \%$ \\
\hline & & $\%$ del total & $44,4 \%$ & $37,8 \%$ & $17,8 \%$ & $100,0 \%$ \\
\hline
\end{tabular}

En cuanto al rendimiento académico y la dimensión Sentido de pertenencia, ambos grupos de estudio presentan un nivel predominantemente Bajo ( $89,3 \%$ y $77.4 \%$ respectivamente). 
Tabla 10

Rendimiento académico y Sentido de pertenencia

\begin{tabular}{|c|c|c|c|c|c|c|}
\hline & & & \multicolumn{3}{|c|}{ Sentido de pertenencia } & \multirow[b]{2}{*}{ Total } \\
\hline & & & Bajo & Medio & Bajo & \\
\hline \multirow[t]{8}{*}{ Rendimiento } & Bajo & Recuento & 25 & 2 & 1 & 28 \\
\hline & & $\%$ dentro de Rendimiento & $89,3 \%$ & $7,1 \%$ & $3,6 \%$ & $100,0 \%$ \\
\hline & & $\%$ dentro de senpert & $34,2 \%$ & $20,0 \%$ & $14,3 \%$ & $31,1 \%$ \\
\hline & & $\%$ del total & $27,8 \%$ & $2,2 \%$ & $1,1 \%$ & $31,1 \%$ \\
\hline & Alto & Recuento & 48 & 8 & 6 & 6 \\
\hline & & $\%$ dentro de Rendimiento & $77,4 \%$ & $12,9 \%$ & $9,7 \%$ & $100,0 \%$ \\
\hline & & $\%$ dentro de senpert & $65,8 \%$ & $80,0 \%$ & $85,7 \%$ & $68,9 \%$ \\
\hline & & $\%$ del total & $53,3 \%$ & $8,9 \%$ & $6,7 \%$ & $68,9 \%$ \\
\hline \multirow[t]{4}{*}{ Total } & & Recuento & 73 & 10 & 7 & 90 \\
\hline & & $\%$ dentro de Rendimiento & $81,1 \%$ & $11,1 \%$ & $7,8 \%$ & $100,0 \%$ \\
\hline & & $\%$ dentro de senpert & $100,0 \%$ & $100,0 \%$ & $100,0 \%$ & $100,0 \%$ \\
\hline & & $\%$ del total & $81,1 \%$ & $11,1 \%$ & $7,8 \%$ & $100,0 \%$ \\
\hline
\end{tabular}

En relación con rendimiento académico y la dimensión Amor familiar, ambos grupos de estudio presentan predominantemente un nivel Bajo $(75 \% \mathrm{y}$ $72,6 \%$ respectivamente).

$\mathrm{Al}$ respecto de los hallazgos con respecto a las dimensiones Sentido de pertenencia y amor Familiar, es necesario considerar que la estudiante universitaria tiene a lo largo de su período de estudio, una carga de trabajos académicos de índole individual y/o grupal que disminuye su tiempo para compartir actividades con la familia, por otro lado en la edad en que se encuentran se busca interactuar con más frecuencia con el grupo de amigos o compañeros de estudios, o con una persona con la que se mantiene una relación amorosa.

Tabla 11

Rendimiento académico y Amor familiar

\begin{tabular}{|c|c|c|c|c|c|c|}
\hline & & & \multicolumn{3}{|c|}{ Amor.Familiar } & \multirow[b]{2}{*}{ Total } \\
\hline & & & Bajo & Medio & Bajo & \\
\hline \multirow[t]{8}{*}{ Rendimiento } & Bajo & Recuento & 21 & 7 & 0 & 28 \\
\hline & & $\%$ dentro de Rendimiento & $75,0 \%$ & $25,0 \%$ & $0,0 \%$ & $100,0 \%$ \\
\hline & & $\%$ dentro de .amor.fam & $31,8 \%$ & $46,7 \%$ & $0,0 \%$ & $31,1 \%$ \\
\hline & & $\%$ del total & $23,3 \%$ & $7,8 \%$ & $0,0 \%$ & $31,1 \%$ \\
\hline & Alto & Recuento & 45 & 8 & 9 & 62 \\
\hline & & $\%$ dentro de Rendimiento & $72,6 \%$ & $12,9 \%$ & $14,5 \%$ & $100,0 \%$ \\
\hline & & $\%$ dentro de amor.fam & $68,2 \%$ & $53,3 \%$ & $100,0 \%$ & $68,9 \%$ \\
\hline & & $\%$ del total & $50,0 \%$ & $8,9 \%$ & $10,0 \%$ & $68,9 \%$ \\
\hline \multirow[t]{4}{*}{ Total } & & Recuento & 66 & 15 & 9 & 90 \\
\hline & & $\%$ dentro de Rendimiento & $73,3 \%$ & $16,7 \%$ & $10,0 \%$ & $100,0 \%$ \\
\hline & & $\%$ dentro de amor.fam & $100,0 \%$ & $100,0 \%$ & $100,0 \%$ & $100,0 \%$ \\
\hline & & $\%$ del total & $73,3 \%$ & $16,7 \%$ & $10,0 \%$ & $100,0 \%$ \\
\hline
\end{tabular}


En cuanto al rendimiento académico y la dimensión de Alegría hallamos que ambos grupos presentan mayoritariamente un nivel Bajo (92,9 y $83.9 \%$ respectivamente).

Tabla 12

Rendimiento académico y Alegría

\begin{tabular}{|c|c|c|c|c|c|c|}
\hline & & & & Alegria & & \\
\hline & & & Bajo & Medio & Bajo & Total \\
\hline Rendimiento & Bajo & Recuento & 26 & 2 & 0 & 28 \\
\hline & & $\%$ dentro de Rendimiento & $92,9 \%$ & $7,1 \%$ & $0,0 \%$ & $100,0 \%$ \\
\hline & & $\%$ dentro de Alegria & $33,3 \%$ & $40,0 \%$ & $0,0 \%$ & $31,1 \%$ \\
\hline & & $\%$ del total & $28,9 \%$ & $2,2 \%$ & $0,0 \%$ & $31,1 \%$ \\
\hline & Alto & Recuento & 52 & 3 & 7 & 62 \\
\hline & & $\%$ dentro de Rendimiento & $83,9 \%$ & $4,8 \%$ & $11,3 \%$ & $100,0 \%$ \\
\hline & & $\%$ dentro de dx.alegria & $66,7 \%$ & $60,0 \%$ & $100,0 \%$ & $68,9 \%$ \\
\hline & & $\%$ del total & $57,8 \%$ & $3,3 \%$ & $7,8 \%$ & $68,9 \%$ \\
\hline Total & & Recuento & 78 & 5 & 7 & 90 \\
\hline & & $\%$ dentro de Rendimiento & $86,7 \%$ & $5,6 \%$ & $7,8 \%$ & $100,0 \%$ \\
\hline & & $\%$ dentro de Alegría & $100,0 \%$ & $100,0 \%$ & $100,0 \%$ & $100,0 \%$ \\
\hline & & $\%$ del total & $86,7 \%$ & $5,6 \%$ & $7,8 \%$ & $100,0 \%$ \\
\hline
\end{tabular}

En relación a rendimiento académico y la dimensión de Tranquilidad, ambos grupos presentan

Tabla 13

Rendimiento académico y Tranquilidad mayoritariamente un nivel Bajo $(85,7 \%$ y $71 ; 0 \%$ respectivamente).

\begin{tabular}{|c|c|c|c|c|c|c|}
\hline & & & \multicolumn{3}{|c|}{ Tranquilidad } & \multirow[b]{2}{*}{ Total } \\
\hline & & & Bajo & Medio & Bajo & \\
\hline \multirow[t]{8}{*}{ Rendimiento } & Bajo & Recuento & 24 & 3 & 1 & 28 \\
\hline & & $\%$ dentro de Rendimiento & $85,7 \%$ & $10,7 \%$ & $3,6 \%$ & $100,0 \%$ \\
\hline & & $\%$ dentro de Tranquilidad & $35,3 \%$ & $18,8 \%$ & $16,7 \%$ & $31,1 \%$ \\
\hline & & $\%$ del total & $26,7 \%$ & $3,3 \%$ & $1,1 \%$ & $31,1 \%$ \\
\hline & Alto & Recuento & 44 & 13 & 5 & 62 \\
\hline & & $\%$ dentro de Rendimiento & $71,0 \%$ & $21,0 \%$ & $8,1 \%$ & $100,0 \%$ \\
\hline & & $\%$ dentro de Tranquilidad & $64,7 \%$ & $81,3 \%$ & $83,3 \%$ & $68,9 \%$ \\
\hline & & $\%$ del total & $48,9 \%$ & $14,4 \%$ & $5,6 \%$ & $68,9 \%$ \\
\hline \multirow[t]{4}{*}{ Total } & & Recuento & 68 & 16 & 6 & 90 \\
\hline & & $\%$ dentro de Rendimiento & $75,6 \%$ & $17,8 \%$ & $6,7 \%$ & $100,0 \%$ \\
\hline & & $\%$ dentro de Tranquilidad & $100,0 \%$ & $100,0 \%$ & $100,0 \%$ & $100,0 \%$ \\
\hline & & $\%$ del total & $75,6 \%$ & $17,8 \%$ & $6,7 \%$ & $100,0 \%$ \\
\hline
\end{tabular}

En cuanto a rendimiento académico y la dimensión Felicidad se halla en ambos grupos que presentan predominantemente un nivel Bajo $(82,1 \%$ y $80,6 \%$ respectivamente).

Con respecto a los hallazgos en las dimensiones Alegría, Tranquilidad y Felicidad en niveles Bajos, es importante señalar que en el período de la adolescencia tardía y juventud, la familia paulatinamente va ocupando un segundo plano en sus sentimientos y vivencias, siendo de mayor preferencia las experiencias que se viven con el grupo de iguales. 
Tabla 14

Rendimiento académico y Felicidad

\begin{tabular}{|c|c|c|c|c|c|c|}
\hline & & & \multicolumn{3}{|c|}{ Felicidad } & \multirow[b]{2}{*}{ Total } \\
\hline & & & Bajo & Medio & Bajo & \\
\hline \multirow[t]{8}{*}{ Rendimiento } & Bajo & Recuento & 23 & 5 & 0 & 28 \\
\hline & & $\%$ dentro de Rendimiento & $82,1 \%$ & $17,9 \%$ & $0,0 \%$ & $100,0 \%$ \\
\hline & & $\%$ dentro de Felicidad & $31,5 \%$ & $38,5 \%$ & $0,0 \%$ & $31,1 \%$ \\
\hline & & $\%$ del total & $25,6 \%$ & $5,6 \%$ & $0,0 \%$ & $31,1 \%$ \\
\hline & Alto & Recuento & 50 & 8 & 4 & 62 \\
\hline & & $\%$ dentro de Rendimiento & $80,6 \%$ & $12,9 \%$ & $6,5 \%$ & $100,0 \%$ \\
\hline & & $\%$ dentro de Felicidad & $68,5 \%$ & $61,5 \%$ & $100,0 \%$ & $68,9 \%$ \\
\hline & & $\%$ del total & $55,6 \%$ & $8,9 \%$ & $4,4 \%$ & $68,9 \%$ \\
\hline \multirow[t]{4}{*}{ Total } & & Recuento & 73 & 13 & 4 & 90 \\
\hline & & $\%$ dentro de Rendimiento & $81,1 \%$ & $14,4 \%$ & $4,4 \%$ & $100,0 \%$ \\
\hline & & $\%$ dentro de Felicidad & $100,0 \%$ & $100,0 \%$ & $100,0 \%$ & $100,0 \%$ \\
\hline & & $\%$ del total & $81,1 \%$ & $14,4 \%$ & $4,4 \%$ & $100,0 \%$ \\
\hline
\end{tabular}

Finalmente, en relación al rendimiento académico y el Total de Satisfacción familiar, ambos grupos

Tabla 15

Rendimiento académico y Total de Satisfacción familiar presentan mayoritariamente un nivel Bajo $(82,1 \% \mathrm{y}$ $82,1 \%$ respectivamente)

\begin{tabular}{|c|c|c|c|c|c|c|}
\hline & & & \multicolumn{3}{|c|}{ Total Satisfacción Familiar } & \multirow[b]{2}{*}{ Total } \\
\hline & & & Bajo & Medio & Bajo & \\
\hline \multirow[t]{8}{*}{ Rendimiento } & Bajo & Recuento & 23 & 5 & 0 & 28 \\
\hline & & $\%$ dentro de Rendimiento & $82,1 \%$ & $17,9 \%$ & $0,0 \%$ & $100,0 \%$ \\
\hline & & $\%$ dentro de TotalSat.Fam & $31,1 \%$ & $45,5 \%$ & $0,0 \%$ & $31,1 \%$ \\
\hline & & $\%$ del total & $25,6 \%$ & $5,6 \%$ & $0,0 \%$ & $31,1 \%$ \\
\hline & Alto & Recuento & 51 & 6 & 5 & 62 \\
\hline & & $\%$ dentro de Rendimiento & $82,3 \%$ & $9,7 \%$ & $8,1 \%$ & $100,0 \%$ \\
\hline & & $\%$ dentro de TotalSat.Fam & $68,9 \%$ & $54,5 \%$ & $100,0 \%$ & $68,9 \%$ \\
\hline & & $\%$ del total & $56,7 \%$ & $6,7 \%$ & $5,6 \%$ & $68,9 \%$ \\
\hline \multirow[t]{4}{*}{ Total } & & Recuento & 74 & 11 & 5 & 90 \\
\hline & & $\%$ dentro de Rendimiento & $82,2 \%$ & $12,2 \%$ & $5,6 \%$ & $100,0 \%$ \\
\hline & & $\%$ dentro de TotalSat. Fam & $100,0 \%$ & $100,0 \%$ & $100,0 \%$ & $100,0 \%$ \\
\hline & & $\%$ del total & $82,2 \%$ & $12,2 \%$ & $5,6 \%$ & $100,0 \%$ \\
\hline
\end{tabular}

\section{CONCLUSIONES:}

- El estilo de aprendizaje Abstracto Secuencial es el que se halla más desarrollado en toda la muestra estudiada.

- El estilo de aprendizaje Concreto Secuencial, en ambos grupos se concentran en un nivel Medio y Alto, siendo el estilo de aprendizaje de mayor preferencia del grupo de estudio.
- La dimensión Holding se halla en un nivel adecuado en el grupo de rendimiento académico Alto.

- La dimensión Sentido de pertenencia, ambos grupos de estudio presentan un nivel predominantemente Bajo.

- La dimensión Amor familiar, en ambos grupos de estudio presentan predominantemente un nivel Bajo. 
- La dimensión de Alegría ambos grupos presentan mayoritariamente un nivel Bajo

- La dimensión de Tranquilidad, ambos grupos presentan mayoritariamente un nivel Bajo.

- La dimensión de Felicidad se halla en ambos grupos mayoritariamente en un nivel Bajo.

- El Total de Satisfacción familiar, ambos grupos presentan mayoritariamente un nivel Bajo.

\section{REFERENCIAS}

Antelm L.,Ana, Gil-López,Alfonso J., Cacheiro-González, María Luz (2015) Análisis del fracaso escolar desde la perspectiva del alumnado y su relación con el estilo de aprendizaje. Educación y Educadores. sep-dic. 18 (3) $471-489$

Bacon, Donald R \& Hartley, Steven W. (2015) Exploring antecedents of performance differences on visual and verbal test items: learning styles versus aptitude. Marketing Education Review. 25 (3) 205-214.

Barraca, J.; López- Yarto (2010) ESFA Escala de satisfacción familiar por adjetivos. Madrid: TEA

Brunton, Bruce (2015) Learning Styles and Student Performance in Introductory Economics. Journal of Education for Business. Feb/Mar, 90 (2). 89-95.

Butler, Kathleen, A. (2000) Performance Based Learning with Style. Columbia, CT: the Learner's Dimension.

Butler, Kathleen A. (1988) Learning Style in Learning. Springhouse Corporation

Chen Kang, L. \& Sidhu, Manjit, S. (2015) Engineering Students Learning Preferences in UNITEN: Comparative Study and Patterns of Learning Styles. Journal of Educational Technology \& Society. Jul, 18 (3) 266-281.

Esguerra Pérez, Gustavo; Guerrero Ospina, Pablo; (2010). Estilos de aprendizaje y rendimiento académico en estudiantes de Psicología. Diversitas: Perspectivas en Psicologíal (1): 97-109.

Guevara Melo, E. P.; Jaramillo Concha,R. Tovar Paredes, S. (2013). Factores familiares y su relación con el rendimiento académico en estudiantes de psicología. Revista Virtual Universidad Católica del Norte. 40 (1)

Guzmán Arteaga, Ramiro, Pacheco Lora, Martha Cecilia (2014) Comunicación familiar y desempeño académico en estudiantes universitarios. Zona
Próxima. ene-jun, 20, 79-91.

Jiménez, C., Aguado., Álvarez, B., Gil, J. A., y Jiménez, R. (2002) Caracterización de los alumnos con Premio Extraordinario de Bachillerato. Bordón, 54 (2-3) ,383-398.

Martínez O. Bertha; Quezada Z. Jenny, Sassone Paul y Huarachi Ch. María E. (2001) Habilidades cognitivas y estilos de aprendizaje. Lima: Biblioteca Nacional del Perú

Nuzhat, A., Salem, O., Hamdan, N., Ashour, N. (2013) Gender differences in learning styles and academic performance of medical students in Saudi Arabia. Medical Teacher. Apr. Supplement, 35, S78-S82.

Quezada J.; Zavala E. Lenti M. (2015) Satisfacción familiar en mujeres jóvenes. Avances en Psicología 23 (2) 223-229

Shankar, P. Ravi, Balasubramanium, Ramanan, Dwivedi, Neelam R., Ramireddy, Ravi,Barton, Byron ( 2014) Learning Styles of Premedical and Basic Science Undergraduate Medical Students in a Caribbean Medical School. Education in Medicine Journal. 6 (3) $58-62$.

Segarra, Mercedes, Estrada, Marta, Monferrer, Diego (2015) Estilos de aprendizaje en estudiantes universitarios: lateralización vs. Interconexión de los hemisferios cerebrales. Revista Española de Pedagogía. sep-dic. 73 (262). 583-600.

Schmeck,R (1988) Learning strategies and learning styles. New York; Plenum Pres.

Tan, Lin Mei \& Laswad, Fawzi (2015) Academic Performance in Introductory Accounting: Do Learning Styles Matter? Accounting Education. Oct, 24 (5). 383-402.

Uribe Delgado, Hugo; Soares Formiga, Nilton; Omar, Alicia; Urteaga, Alicia Florencia; (2010). Capital sociocultural familiar, autoestima y desempeño académico en adolescentes. Revista Latinoamericana de Estudios Educativos, Sin mes, 93-114.

Terregrossa, R. A. , Englander, F. , Zhaobo W. (2015) Learning style theory as a potential tool in guiding student choice of college major. International Journal of Education Research. Spring, 10 (1) 24-38.

Williams, B., Brown, T., Etherington, J. (2013) Learning Style Preferences of Undergraduate Social Work Students. Social Work Education. Dec, 32 ( 8) 972-990

Fecha de recepción: 1 de octubre, 2019

Fecha de aceptación: 20 de octubre, 2019 